\title{
The Mosaic of “Seronegative” Antiphospholipid Syndrome
}

\author{
Fabrizio Conti, ${ }_{1}^{1}$ Antonella Capozzi, ${ }^{2}$ Simona Truglia, ${ }^{1}$ Emanuela Lococo, ${ }^{2}$ Agostina Longo, ${ }^{2}$ \\ Roberta Misasi, ${ }^{2}$ Cristiano Alessandri, ${ }^{1}$ Guido Valesini, ${ }^{1}$ and Maurizio Sorice ${ }^{2}$ \\ ${ }^{1}$ Reumatologia, Dipartimento di Medicina Interna e Specialità Mediche, Sapienza Università di Roma, \\ Viale del Policlinico 155, 00161 Roma, Italy \\ ${ }^{2}$ Dipartimento di Medicina Sperimentale, Sapienza Università di Roma, Viale Regina Elena 324, 00161 Roma, Italy
}

Correspondence should be addressed to Maurizio Sorice; maurizio.sorice@uniromal.it

Received 13 December 2013; Accepted 10 February 2014; Published 17 March 2014

Academic Editor: Michael Mahler

Copyright (C) 2014 Fabrizio Conti et al. This is an open access article distributed under the Creative Commons Attribution License, which permits unrestricted use, distribution, and reproduction in any medium, provided the original work is properly cited.

\begin{abstract}
In the clinical practice it is possible to find patients with clinical signs suggestive of antiphospholipid syndrome (APS), who are persistently negative for the laboratory criteria of APS, that is, anti-cardiolipin antibodies (aCL), anti- $\beta_{2}$-GPI antibodies and lupus anticoagulant. Therefore, it was proposed for these cases the term of seronegative APS (SN-APS). In order to detect autoantibodies with different methodological approaches, sera from 24 patients with SN-APS were analysed for anti-phospholipid antibodies using TLC immunostaining, for anti-vimentin/cardiolipin antibodies by enzyme-linked immunosorbent assay (ELISA), and for antiannexin V and anti-prothrombin antibodies by ELISA and dot blot. Control groups of our study were 25 patients with APS, 18 with systemic lupus erythematosus (SLE), and 32 healthy controls. Results revealed that 13/24 (54.2\%) SN-APS sera were positive for aCL ( 9 of whom were also positive for lysobisphosphatidic acid) by TLC immunostaining, 11/24 (45.8\%) for anti-vimentin/cardiolipin antibodies, $3 / 24$ (12.5\%) for anti-prothrombin antibodies, and 1/24 (4.2\%) for anti-annexin $\mathrm{V}$ antibodies. These findings suggest that in sera from patients with SN-APS, antibodies may be detected using "new" antigenic targets (mainly vimentin/cardiolipin) or methodological approaches different from traditional techniques (mainly TLC immunostaining). Thus, SN-APS represents a mosaic, in which antibodies against different antigenic targets may be detected.
\end{abstract}

\section{Introduction}

Antiphospholipid antibody syndrome (APS) is characterized by arterial and/or venous thromboses, recurrent abortions or foetal loss, and circulating antiphospholipid antibodies (aPL) $[1,2]$. According to the classification criteria, diagnosis of APS requires the combination of at least one clinical and one laboratory criterion $[3,4]$. Anti-CL and anti- $\beta_{2}-$ glycoprotein-I $\left(\mathrm{a} \beta_{2}\right.$-GPI) antibodies, detected by enzyme linked immunosorbent assay (ELISA) and the lupus anticoagulant (LA) detected by clotting assays are the recommended tests for the detection of aPL $[5,6]$. Nevertheless, in daily clinical practice it is possible to find patients with clinical signs suggestive of APS who are persistently negative for the routinely used aCL, a $\beta_{2}$-GPI, and LA. Therefore, it was proposed for these cases the term of "seronegative APS" (SNAPS) $[2,7]$. Three possible explanations for the existence of such "seronegative" cases have been proposed: either the diagnosis is wrong, or that previously positive aPL tests have become negative, or, as seems most likely, the current range of tests is inadequate [8].

Indeed, aPL represent a heterogeneous family of antibodies reacting with phospholipid-binding cofactor proteins, including not only $\beta_{2}$-GPI $[9,10]$ but also different anionic phospholipids, proteins, or phospholipid-protein complexes, such as prothrombin [11], protein S [12, 13], protein C [14], annexin V [15], annexin II [16], oxidized low-density lipoprotein, lysobisphosphatidic acid (LBPA), and sulfatides [17-19].

Recently, with a proteomic approach, we identified vimentin/cardiolipin as a "new" target of the APS, also detectable in SN-APS patients [20]. In addition, we demonstrated the possibility of detecting aPL in SN-APS patients by immunostaining on thin-layer chromatography (TLC) plates $[21,22]$. This latter nonquantitative technique identifies the 
reactivity of serum aPL with phospholipid molecules but with a different antigenic exposure as compared to ELISA method.

The aim of this study was to identify the best screening combination of "new" antigenic targets or methodological approaches to detect aPL in SN-APS patients.

\section{Materials and Methods}

2.1. Patients. The study included 24 consecutive patients, attending the Lupus clinic, Rheumatology Unit of the Sapienza University of Rome, presenting clinical features consistent with a diagnosis of APS but tested persistently negative (at least 2 times 12 weeks apart) for conventional aCL, a $\beta_{2}$-GPI, and LA tests. Clinical manifestations included venous and/or arterial thrombosis and pregnancy morbidity as stated in the classification criteria for definite APS [3, 4]. Sera were collected at several times and stored at $-20^{\circ} \mathrm{C}$ until use. Moreover, all patients showed normal screening for other causes of thrombophilia such as antithrombin, protein $\mathrm{C}$ and protein $\mathrm{S}$ deficiency, hyperhomocysteinemia, factor $\mathrm{V}$, and prothrombin mutations.

Moreover, in this study, 43 consecutive out-patients, attending the Rheumatology Division of Sapienza University of Rome, were also included. Twenty-five had APS, diagnosed according to the Sapporo criteria [4]; they included both primary APS $(N=9)$ and APS associated with SLE $(N=$ 16); 18 had SLE fulfilling the ACR revised criteria for the classification of SLE [23]. Finally 32 healthy subjects (normal blood donors) matched for age and sex were studied as controls.

This study was approved by the local ethic committees and participants gave written informed consent.

2.2. ELISA for aPL and Antiphospholipid-Binding Proteins. aCL and a $\beta_{2}$-GPI ELISA kits were obtained from INOVA Diagnostics Inc. (San Diego, CA, USA). ELISA was performed according to the manufacturer's instructions. Antiannexin $\mathrm{V}$ and antiprothrombin were performed as previously described [11, 15] and confirmed by dot blot. A positive control and several normal human sera were run in the same assay to confirm the specificity of the results.

2.3. LA Test. LA was studied in two coagulation systems, a dilute sensitized activated partial thromboplastin time (aPTT) and a dilute Russell's viper venom time (dRVVT), followed by confirm test, using reagents and instrumentation by Hemoliance Instrumentation Laboratory, Lexington, MA, USA.

2.4. Detection of aPL by TLC Immunostaining. Immunostaining on TLC was performed as previously described, with slight modification [21, 22, 24, 25]. CL (Sigma Chemical Co., St Louis, MO, USA) and LBPA (Avanti Polar Lipids, Alabaster, AL, USA), $2 \mu \mathrm{g}$, were run on aluminium-backed silica gel $60(20 \times 20)$ high performance thin layer chromatography (HPTLC) plates (Merck Co, Inc Darmstadt, Germany). Chromatography was performed in chloroform : methanol: $\mathrm{CH}_{3} \mathrm{COOH}$ : water $(100: 75: 7: 4)$ $(\mathrm{v}: \mathrm{v}: \mathrm{v}: \mathrm{v})$. The dried chromatograms were soaked for $90 \mathrm{sec}$ in a $0.5 \%(\mathrm{w}: \mathrm{v})$ solution of poly(isobutyl methacrylate) beads (Polysciences, Inc. Eppelheim, Germany) dissolved in hexane. After air-drying, the chromatograms were incubated at room temperature for $1 \mathrm{~h}$ with $1 \%$ BSA in PBS to eliminate nonspecific binding. The blocking solution was removed and replaced by a washing buffer (phosphate-buffered saline, PBS). The chromatograms were then incubated for $1 \mathrm{~h}$ at room temperature with sera, diluted 1:100 in the blocking solution. Sera were removed and chromatograms were washed 3 times for $10 \mathrm{~min}$ with PBS. Bound antibodies were visualized with HRP-conjugated goat anti-human IgG (Sigma-Aldrich, St Louis, MO, USA), diluted 1:1000 in 1\% bovine serum albumin (BSA) in PBS, and incubated at room temperature for $1 \mathrm{~h}$ and immunoreactivity was assessed by the chemiluminescence reaction using the ECL Western blotting system (Amersham Pharmacia Biotech, Buckinghamshire, UK).

2.5. Detection of Antivimentin/Cardiolipin by ELISA. Antivimentin/cardiolipin complex antibodies were detected by a slight modification of ELISA method previously reported [20]. Polystyrene plates (96-well) were coated and incubated overnight at $4^{\circ} \mathrm{C}$ with $100 \mu \mathrm{L} /$ well of cardiolipin $(50 \mu \mathrm{g} / \mathrm{mL}$; Sigma-Aldrich) in methanol and then with $100 \mu \mathrm{L} /$ well of human recombinant vimentin $(5 \mu \mathrm{g} / \mathrm{mL}$; R\&D System, Minneapolis, MN, USA) in $0.05 \mathrm{M} \mathrm{NaHCO}$ buffer, $\mathrm{pH}$ 9.5. Coated plates were incubated overnight at $4^{\circ} \mathrm{C}$ and then washed 3 times with phosphate-buffered saline containing $0.1 \%$ Tween 20 (PBS-T). Plates were blocked for $2 \mathrm{~h}$ at room temperature with $100 \mu \mathrm{L}$ of $1 \%$ BSA in PBS. After washing 3 times with PBS-T, the wells were incubated for $1 \mathrm{~h}$ at room temperature, with $100 \mu \mathrm{L}$ of patient sera, diluted $1: 100$ in the blocking buffer. Each serum was analyzed in triplicate. Goat polyclonal antivimentin (R\&D Systems) was used as a positive control.

After 3 washes with PBS-T, the plates were incubated for $1 \mathrm{~h}$ at room temperature with horseradish peroxidaseconjugated antibodies; either goat anti-human IgG or rabbit anti-goat IgG (Sigma-Aldrich) was diluted in 1\% BSA in PBS. The plates were washed 3 times with PBS-T; the bound peroxidase was then revealed with $100 \mu \mathrm{L}$ of $O$-phenylenediamine dihydrochloride and color development was stopped with $\mathrm{H}_{2} \mathrm{SO}_{4} 0.2 \mathrm{M}$ for $5 \mathrm{~min}$. Absorbance was measured at $492 \mathrm{~nm}$ in a microplate reader. Data were presented as the mean optical density (OD) corrected for background (wells without coated antigen). Thirty-two normal human sera were also tested, and a cut-off value was established at a mean of optical density (OD) \pm 3 SD of normal human sera. Parallel experiments were performed in which all the procedures were identical without coated vimentin/cardiolipin complex. Virtually no reactivity was detected in all the samples (data not shown).

\section{Results}

3.1. Characteristics of Patients. All but one (a 39-year-old female with Asian ethnicity) SN-APS patients enrolled in this 
TABLE 1: Clinical characteristics of patients studied.

\begin{tabular}{lcc}
\hline Characteristics $n$ (\%) & $\begin{array}{c}\text { SN-APS } \\
(n=24)\end{array}$ & $\begin{array}{c}\text { APS } \\
(n=25)\end{array}$ \\
\hline Other autoimmune diseases & $17(70.8)$ & $16(64)$ \\
SLE & $9(37.5)$ & $16(64)$ \\
Sjögren syndrome secondary to SLE & $2(8.3)$ & $8(32)$ \\
Primary Sjögren syndrome & $1(4.2)$ & 0 \\
Rhupus & $1(4.2)$ & 0 \\
Mixed connective tissue disease & $1(4.2)$ & 0 \\
Undifferentiated connective tissue & $1(4.2)$ & 0 \\
Disease & $1(4.2)$ & 0 \\
Urticarial vasculitis & $1(4.2)$ & 0 \\
LED & $1(4.2)$ & 0 \\
Myasthenia gravis & 0 & $1(4)$ \\
None & $5(20.8)$ & $9(36)$ \\
Vascular thrombosis & $18(75)$ & $24(96)$ \\
Venous thrombosis & $10(41.6)$ & $17(68)$ \\
Arterial thrombosis & $12(50)$ & $10(40)$ \\
Recurrent thrombosis & $8(33.3)$ & $10(40)$ \\
Pregnancy morbidity & $8(33.3)$ & $9(36)$ \\
Normal fetus deaths & $7(29.2)$ & $2(8)$ \\
Premature births & 0 & 0 \\
Spontaneous abortions & $5(20.8)$ & $8(32)$ \\
Vascular thrombosis and pregnancy morbidity & $3(12.5)$ & $6(24)$ \\
Noncriteria APS features & $12(5.3)$ & $2(8)$ \\
Livedo reticularis & $5(20.8)$ & $7(28)$ \\
Thrombocytopenia & $1(4.2)$ & $5(20)$ \\
Cognitive dysfunctions & $1(4.2)$ & $6(24)$ \\
Migraine & $5(20.8)$ & $8(32)$ \\
Seizures & 0 & $5(20)$ \\
Hrain MRI scan abnormalities & $8(32)$ & $14(56)$ \\
Smoketes mellitus & $10(40)$ \\
Hypertension & $5(20)$ \\
\hline
\end{tabular}

study were Caucasian, 18 females and 6 males with a mean age of 45.7 years (range 26-82), and a mean disease duration of 9.3 years (range $0.8-57$ ). The clinical characteristics of SNAPS patients are reported in Table 1. APS patients ( 3 males and 22 females) showed a mean age of 43.9 years (range 2771 ) and a mean disease duration of 9.6 years (range $0.1-34$ ). SLE patients were all females with a mean age of 36.8 years (range 18-59) and a mean disease duration of 13.4 years (range 0.8-36). None of the healthy subjects experienced arterial or venous thrombosis nor recurrent fetal loss.

A statistically significant correlation was found between arterial and/or venous thrombosis and pregnancy morbidity in SN-APS $(P<0.001)$.
3.2. Detection of $a P L$ by TLC Immunostaining. In SN-APS patients the results obtained by TLC immunostaining showed the presence of $\mathrm{aCl}$ in 13 out of 24 patients (54.2\%); anti-LBPA antibodies in 9 out of 24 (37.5\%). All sera positive for antiLBPA were also positive for aCL.

In APS patients TLC immunostaining showed the presence of antibodies against CL in 17/25 (68\%), against LBPA in 14/25 (56\%). In SLE patients TLC immunostaining showed the presence of antibodies against CL in 11/18 (61.1\%), against LBPA in 11/18 (61.1\%). Finally, none of the healthy subjects showed aPL reactivity by TLC immunostaining (Table 2 ).

3.3. Detection of aPL and Antiphospholipid-Binding Proteins by ELISA. Eleven out of 24 SN-APS patients (45.8\%) showed serum antibodies (IgG class) against vimentin/cardiolipin, 3 (12.5\%) against prothrombin, and 1 (4.2\%) against annexin V (Table 2). None resulted positive for antibodies against CL or $\beta_{2}$-GPI.

In APS patients anti-vimentin/CL antibodies were detected in 22/25 (88\%), antiprothrombin in 9/25 (36\%), and antiannexin $\mathrm{V}$ in $14 / 25$ (56\%). Anti-CL reactivity was observed in $25 / 25(100 \%)$. Anti- $\beta_{2}$-GPI reactivity was observed in 18/25 (72\%) APS.

In SLE patients anti-vimentin/CL antibodies were detected in 7/18 (38.8\%), anti-prothrombin in 1/18 (5.5\%), and antiannexin $\mathrm{V}$ in $4 / 18(22.2 \%)$. Moreover, anti-CL reactivity was observed in $14 / 18(77.7 \%)$ and anti- $\beta_{2}$-GPI reactivity was observed in 7/18 (38.8\%) SLE patients.

Finally, none of the 32 healthy subjects displayed positivity for the autoantibodies under test.

3.4. Associations between Autoantibodies and Clinical Features. Taken together, our findings show that in 19 out of 24 SN-APS (79.2\%) at least one aPL/cofactor antibody was detected using the assays under test (Table 3 ).

Table 4 shows the prevalence of the autoantibodies in SN-APS patients with different clinical manifestations. No significant association was found between the prevalence of the clinical features in SN-APS patients and specific autoantibodies. The combination of two of the tested methodological approaches, TLC immunostaining for aCL and ELISA for anti-vimentin/cardiolipin complex antibodies, was able to detect aPL/cofactors in about two-thirds of SN-APS patients with thrombosis or pregnancy morbidity, with a small additional gain when also performing ELISA for prothrombin and annexin V (Table 4).

\section{Discussion}

In the clinical practice it is possible to find patients with clinical signs suggestive of APS, who are persistently negative for the routinely used assays to detect aCL, a $\beta_{2}$-GPI and LA. For these cases the term of SN-APS has been proposed [2630]. Similarly to classical APS, SN-APS can have an accelerated progression, resulting in multiorgan failure, ending to catastrophic APS [31].

Thus, since clinical features of SN-APS appear to be similar to APS, the most convincing explanation for the 
TABLE 2: Occurrence of autoantibodies in SN-APS and control sera.

\begin{tabular}{lcccc}
\hline Autoantibodies & SN-APS (24) $n(\%)$ & APS (25) $n(\%)$ & SLE (18) $n(\%)$ & Healthy donors (32) $n(\%)$ \\
\hline Anticardiolipin by TLC-immunostaining & $13(54.2)$ & $17(68)$ & $11 / 18(61.1)$ & $0(0)$ \\
Antivimentin/Cardiolipin & $11(45.8)$ & $22(88)$ & $7 / 18(38.8)$ & $0(0)$ \\
Antiprothrombin & $3(12.5)$ & $9(36)$ & $1 / 18(5.5)$ & $0(0)$ \\
Antiannexin V & $1(4.2)$ & $14(56)$ & $4 / 18(22.2)$ & $0(0)$ \\
\hline
\end{tabular}

TABLE 3: Positivity of autoantibodies in the 24 SN-APS sera.

\begin{tabular}{|c|c|c|c|c|}
\hline Patient $n$ & aCL by TLC immunostaining & Antivimentin/CL & Antiprothrombin & Antiannexin V \\
\hline 1 & Pos & Pos & Neg & Neg \\
\hline 2 & Pos* & Pos & $\mathrm{Neg}$ & Neg \\
\hline 3 & Pos $^{*}$ & Pos & Neg & $\mathrm{Neg}$ \\
\hline 4 & Pos $^{*}$ & $\mathrm{Neg}$ & Neg & $\mathrm{Neg}$ \\
\hline 5 & Pos & Pos & Neg & Neg \\
\hline 6 & Pos $^{*}$ & Neg & Neg & Neg \\
\hline 7 & Pos $^{*}$ & Neg & Neg & Neg \\
\hline 8 & Pos & Pos & Neg & Neg \\
\hline 9 & Pos* & Pos & Neg & $\mathrm{Neg}$ \\
\hline 10 & Pos & Neg & Neg & Neg \\
\hline 11 & Pos $^{*}$ & Pos & Neg & $\mathrm{Neg}$ \\
\hline 12 & $\operatorname{Pos}^{*}$ & Neg & Neg & Neg \\
\hline 13 & Pos $^{*}$ & Neg & Neg & $\mathrm{Neg}$ \\
\hline 14 & $\mathrm{Neg}$ & $\mathrm{Neg}$ & $\mathrm{Neg}$ & $\mathrm{Neg}$ \\
\hline 15 & $\mathrm{Neg}$ & $\mathrm{Neg}$ & Pos & Pos \\
\hline 16 & $\mathrm{Neg}$ & $\mathrm{Neg}$ & Pos & $\mathrm{Neg}$ \\
\hline 17 & $\mathrm{Neg}$ & Pos & Pos & $\mathrm{Neg}$ \\
\hline 18 & $\mathrm{Neg}$ & $\mathrm{Neg}$ & $\mathrm{Neg}$ & $\mathrm{Neg}$ \\
\hline 19 & $\mathrm{Neg}$ & Pos & $\mathrm{Neg}$ & $\mathrm{Neg}$ \\
\hline 20 & Neg & Pos & Neg & $\mathrm{Neg}$ \\
\hline 21 & Neg & Pos & $\mathrm{Neg}$ & $\mathrm{Neg}$ \\
\hline 22 & $\mathrm{Neg}$ & Neg & $\mathrm{Neg}$ & $\mathrm{Neg}$ \\
\hline 23 & $\mathrm{Neg}$ & $\mathrm{Neg}$ & $\mathrm{Neg}$ & $\mathrm{Neg}$ \\
\hline 24 & $\mathrm{Neg}$ & $\mathrm{Neg}$ & $\mathrm{Neg}$ & $\mathrm{Neg}$ \\
\hline
\end{tabular}

${ }^{*}$ Also positive for anti-LBPA.

TABLE 4: Autoantibody prevalence in seronegative antiphospholipid syndrome (SN-APS) patients $(n=24)$ according to the clinical manifestations.

\begin{tabular}{|c|c|c|c|c|c|}
\hline Autoantibodies (assay) & $\begin{array}{c}\text { Total } \\
\text { thrombosis } \\
(n=18)\end{array}$ & $\begin{array}{c}\text { Arterial } \\
\text { thrombosis } \\
(n=12)\end{array}$ & $\begin{array}{c}\text { Venous } \\
\text { thrombosis } \\
(n=10)\end{array}$ & $\begin{array}{l}\text { Recurrent } \\
\text { thrombosis } \\
(n=8)\end{array}$ & $\begin{array}{c}\text { Pregnancy } \\
\text { morbidity } \\
(n=8)\end{array}$ \\
\hline aCL (TLC) & $11(61.1 \%)$ & $7(58.3 \%)$ & $6(60.0 \%)$ & $5(62.5 \%)$ & $3(37.5 \%)$ \\
\hline Antivimentin/CL & $7(38.9 \%)$ & $4(33.3 \%)$ & $5(50.0 \%)$ & $3(37.5 \%)$ & $5(62.5 \%)$ \\
\hline aCL (TLC) + antivimentin/CL & $12(66.7 \%)$ & $8(66.7 \%)$ & $6(60.0 \%)$ & $5(62.5 \%)$ & $5(62.5 \%)$ \\
\hline Antiannexin V & 0 & 0 & 0 & 0 & $1(12.5 \%)$ \\
\hline Antiprothrombin & $1(5.6 \%)$ & 0 & $1(10.0 \%)$ & $1(12.5 \%)$ & $2(25.0 \%)$ \\
\hline $\begin{array}{l}\text { aCL }(\mathrm{TLC})+\text { antivimentin/CL }+ \text { anti-annexin } \mathrm{V}+ \\
\text { antiprothrombin }\end{array}$ & $13(72.2 \%)$ & $8(66.7 \%)$ & $7(70.0 \%)$ & $6(75.0 \%)$ & $7(87.5 \%)$ \\
\hline No autoantibodies & $5(27.8 \%)$ & $4(33.3 \%)$ & $3(30.0 \%)$ & $2(25.0 \%)$ & $1(12.5 \%)$ \\
\hline
\end{tabular}


existence of such "seronegative" patients may be that the current range of tests is inadequate. It may depend either on limits of the traditional technical approaches or on the existence of different antigenic targets.

Considering the first possibility, we employed a different methodological approach for detection of aPL, TLC immunostaining, which relies upon the different partition characteristics of phospholipids between the surface (stationary phase) and mobile solvent phase for different solvent polarities. TLC immunostaining is useful for detection of aPL in the presence of cofactor proteins, mainly $\beta_{2}$-GPI (provided with the medium). However, in this case, the binding of phospholipid to solid phase mainly involves both electrostatic and hydrophobic interactions. Thus, the antigen exposure is quite different as compared to that on the surface of microtitre wells, where phospholipids are coated in a layer of immobilized lamellar phospholipids [32]. Indeed, the present results obtained by TLC immunostaining showing the presence of aCL in more than half SN-APS patients (54.2\%) confirm our previous reports [22] and suggest that this method may represent a useful tool to detect aCL, mainly in SN-APS patients. Moreover, all sera positive for anti-LBPA by TLC were also positive for CL. A possible limit of this method could be a relatively low sensitivity, since only $68 \%$ of "true" APS sera were positive for aCL by TLC.

Moreover, we have to consider that aPL represent a very heterogeneous family of antibodies because more than 30 different antibodies have been described in APS patients (the so-called autoantibody explosion in antiphospholipid syndrome) [33]. Among them, several "nonclassical" aPL are directed against platelets, glycoproteins, coagulation factors, lamins, mitochondrial antigens, and other cell surface markers [19]. With the aim to discover "new" antigenic targets of aPL, we identified, with a proteomic approach, vimentin/CL as a potential autoantigen in SN-APS patients [20]. In the present study we found serum antibodies against vimentin/CL in about $45 \%$ of SN-APS patients. Interestingly, these latter antibodies were detected in 4 SN-APS patients who were also negative for aCL by TLC immunostaining, supporting the view that antivimentin/CL test may be a relevant additional test for identification of aPL positive patients. Finally, we analyzed the SN-APS sera for the presence of antibodies against two major cofactor proteins for aPL, prothrombin and annexin V. Our results revealed that 3 out of 24 sera from SN-APS patients (12.5\%) displayed antibodies to prothrombin and $1(4.2 \%)$ to annexin V. The datum of antiprothrombin might be underestimated, since we tested antiprothrombin antibodies in the absence of phosphatidylserine. Indeed, anti-phosphatidylserine/prothrombin antibodies, which have been recently standardized and validated [34], seemed to represent a stronger risk factor for thrombosis than antiprothrombin [35]. However, interestingly, in SNAPS group, anti-prothrombin antibodies were detected in 3 patients, which were negative for aCL by TLC immunostaining.

Taken together, these findings indicate that the execution of all these tests (TLC immunostaining, antivimentin/CL, antiprothrombin, and antiannexin $\mathrm{V}$ ) can be very useful for identification of autoantibodies in so-called SN-APS patients.

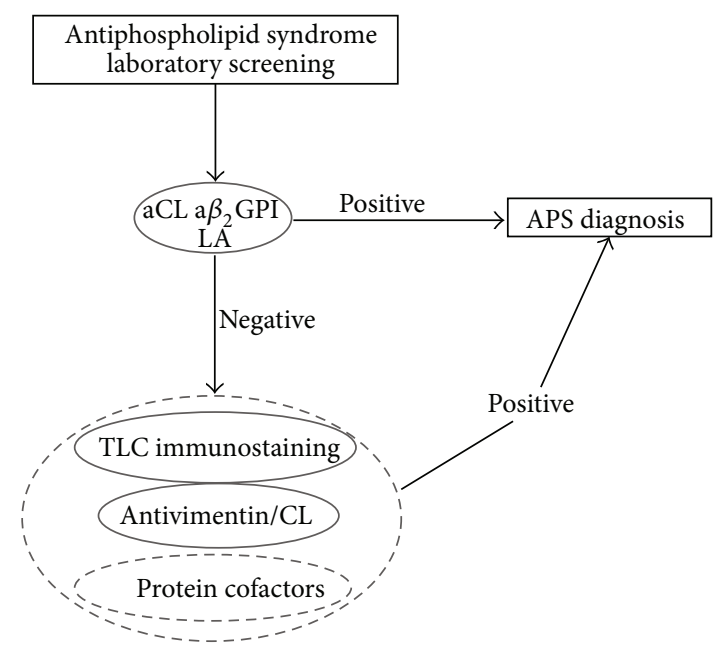

Figure 1: Proposed algorithm depicting the different diagnostic approaches in the most reliable sequence to reveal the hidden reactivity of the conventional aPL detection.

We can assert that, by using these approaches, it is possible to detect autoantibodies in the majority of the patients (79.2\%). We suggest using all these different diagnostic approaches in the most reliable sequence, starting with TLC immunostaining, then antivimentin/CL ELISA, and eventually anticofactors ELISA to reveal the hidden reactivity of the conventional aPL detection (Figure 1). The combination of the first two diagnostic tests, TLC immunostaining and anti-vimentin/CL ELISA, detects aPL in about two-thirds of tested samples.

The presence of these antibodies has several implications. They might be used for early diagnosis of the syndrome, especially among patients at risk for thrombotic events and/or pregnancy morbidity. Moreover, autoantibody presence or absence might subclassify APS according to the association of these antibodies with clinical manifestations. At the end, they may help to identify patients who need secondary thromboprophylaxis with long-term anticoagulation, as well as prophylactic treatment during pregnancy.

However, although these approaches ameliorate our diagnostical possibilities, we are still unable to detect autoantibodies in a percentage of patients with a clinical picture suggestive of SN-APS. Other unidentified cofactors may be involved in sera reactivity. Further studies will shed light on "new" antigenic specificities in SN-APS as well as on the real importance of nonclassical antibodies found in SN-APS.

\section{Conclusions}

These findings suggest that in sera from patients with SNAPS, antibodies may be detected using "new" antigenic targets (mainly vimentin/cardiolipin) or methodological approaches different from traditional techniques (mainly TLC immunostaining). Thus, SN-APS represents a mosaic, in which antibodies against different antigenic targets may be detected. 


\section{Conflict of Interests}

The authors declare that there is no conflict of interests regarding the publication of this paper.

\section{Authors' Contribution}

Fabrizio Conti and Antonella Capozzi equally contributed to this paper.

\section{References}

[1] G. R. V. Hughes, “The anticardiolipin syndrome," Clinical and Experimental Rheumatology, vol. 3, no. 4, pp. 285-286, 1985.

[2] G. R. Hughes, E. N. Harris, and A. E. Gharavi, "The anticardiolipin syndrome," The Journal of Rheumatology, vol. 13, no. 3, pp. 486-489, 1986.

[3] W. A. Wilson, A. E. Gharavi, T. Koike et al., "International consensus statement on preliminary classification criteria for definite antiphospholipid syndrome," Arthritis and Rheumatism, vol. 42, no. 7, pp. 1309-1311, 1999.

[4] S. Miyakis, M. D. Lockshin, T. Atsumi et al., "International consensus statement on an update of the classification criteria for definite antiphospholipid syndrome (APS)," Journal of Thrombosis and Haemostasis, vol. 4, no. 2, pp. 295-306, 2006.

[5] A. Tincani, G. Balestrieri, L. Spatola, M. Cinquini, P. L. Meroni, and R. A. S. Roubey, "Anticardiolipin and anti- $\beta 2$ glycoprotein I immunoassays in the diagnosis of antiphospholipid syndrome," Clinical and Experimental Rheumatology, vol. 16, no. 4, pp. 396402, 1998

[6] M. L. Bertolaccini, S. Gomez, J. F. P. Pareja et al., "Antiphospholipid antibody tests: spreading the net," Annals of the Rheumatic Diseases, vol. 64, no. 11, pp. 1639-1643, 2005.

[7] R. Nayfe, I. Uthman, J. Aoun, E. Saad Aldin, M. Merashli, and M. A. Khamashta, "Seronegative antiphospholipid syndrome," Rheumatology, vol. 52, no. 8, pp. 1358-1367, 2013.

[8] G. R. V. Hughes, "Antiphospholipid syndrome (Hughes syndrome): 10 clinical topics," Lupus, vol. 19, no. 4, pp. 343-346, 2010.

[9] M. Galli, T. Barbui, F. A. Zwaal, P. Comfurius, and E. M. Bevers, "Antiphospholipid antibodies': involvement of protein cofactors," Haematologica, vol. 78, no. 1, pp. 1-4, 1993.

[10] H. B. de Laat, R. H. W. M. Derksen, and P. G. de Groot, “ $\beta 2-$ Glycoprotein I, the playmaker of the antiphospholipid syndrome," Clinical Immunology, vol. 112, no. 2, pp. 161-168, 2004.

[11] J. Arvieux, L. Darnige, C. Caron, G. Reber, J. C. Bensa, and M. G. Colomb, "Development of an ELISA for autoantibodies to prothrombin showing their prevalence in patients with lupus anticoagulants," Thrombosis and Haemostasis, vol. 74, no. 4, pp. 1120-1125, 1995.

[12] M. Sorice, P. Arcieri, T. Griggi et al., "Inhibition of protein S by autoantibodies in patients with acquired protein S deficiency," Thrombosis and Haemostasis, vol. 75, no. 4, pp. 555-559, 1996.

[13] M. Sorice, T. Griggi, A. Circella et al., "Protein S antibodies in acquired protein S deficiencies," Blood, vol. 83, no. 8, pp. 23832384, 1994.

[14] J. D. Oosting, R. H. W. M. Derksen, I. W. G. Bobbink, T. M. Hackeng, B. N. Bouma, and P. G. de Groot, "Antiphospholipid antibodies directed against a combination of phospholipids with prothrombin, protein $\mathrm{C}$, or protein $\mathrm{S}$ : an explanation for their pathogenic mechanism?" Blood, vol. 81, no. 10, pp. 2618$2625,1993$.

[15] J. Kaburaki, M. Kuwana, M. Yamamoto, S. Kawai, and Y. Ikeda, "Clinical significance of anti-annexin $\mathrm{V}$ antibodies in patients with systemic lupus erythematosus," The American Journal of Hematology, vol. 54, no. 3, pp. 209-213, 1997.

[16] V. Salle, J. C. Mazière, A. Smail et al., "Anti-annexin II antibodies in systemic autoimmune diseases and antiphospholipid syndrome," Journal of Clinical Immunology, vol. 28, no. 4, pp. 291-297, 2008.

[17] T. Kobayashi, E. Stang, K. S. Fang, P. de Moerloose, R. G. Parton, and J. Gruenberg, "A lipid associated with the antiphospholipid syndrome regulates endosome structure and function," Nature, vol. 392, no. 6672, pp. 193-197, 1998.

[18] C. Alessandri, M. Bombardieri, L. Di Prospero et al., "Antilysobisphosphatidic acid antibodies in patients with antiphospholipid syndrome and systemic lupus erythematosus," Clinical and Experimental Immunology, vol. 140, no. 1, pp. 173-180, 2005.

[19] G. Valesini and C. Alessandri, "New facet of antiphospholipid antibodies," Annals of the New York Academy of Sciences, vol. 1051, pp. 487-497, 2005.

[20] E. Ortona, A. Capozzi, T. Colasanti et al., "Vimentin/cardiolipin complex as a new antigenic target of the antiphospholipid syndrome," Blood, vol. 116, no. 16, pp. 2960-2967, 2010.

[21] M. Sorice, T. Griggi, A. Circella et al., "Detection of antiphospholipid antibodies by immunostaining on thin layer chromatography plates," Journal of Immunological Methods, vol. 173, no. 1, pp. 49-54, 1994.

[22] F. Conti, C. Alessandri, M. Sorice et al., "Thin-layer chromatography immunostaining in detecting anti-phospholipid antibodies in seronegative anti-phospholipid syndrome," Clinical and Experimental Immunology, vol. 167, no. 3, pp. 429-437, 2012.

[23] M. C. Hochberg, "Updating the American College of Rheumatology revised criteria for the classification of systemic lupus erythematosus," Arthritis and Rheumatism, vol. 40, no. 9, p. $1725,1997$.

[24] C. Alessandri, M. Sorice, M. Bombardieri et al., "Antiphospholipid reactivity against cardiolipin metabolites occurring during endothelial cell apoptosis," Arthritis Research and Therapy, vol. 8, no. 6, article R180, 2006.

[25] M. Sorice, V. Pittoni, T. Griggi et al., "Specificity of antiphospholipid antibodies in infectious mononucleosis: a role for anti-cofactor protein antibodies," Clinical and Experimental Immunology, vol. 120, no. 2, pp. 301-306, 2000.

[26] G. R. V. Hughes and M. A. Khamashta, "Seronegative antiphospholipid syndrome," Annals of the Rheumatic Diseases, vol. 62, no. 12, p. 1127, 2003.

[27] C. Alessandri, F. Conti, P. Conigliaro, R. Mancini, L. Massaro, and G. Valesini, "Seronegative autoimmune diseases," Annals of the New York Academy of Sciences, vol. 1173, pp. 52-59, 2009.

[28] R. Cervera, F. Conti, A. Doria, L. Iaccarino, and G. Valesini, "Does seronegative antiphospholipid syndrome really exist?" Autoimmunity Reviews, vol. 11, no. 8, pp. 581-584, 2012.

[29] M. L. Bertolaccini, M. A. Khamashta, and G. R. V. Hughes, "Diagnosis of antiphospholipid syndrome," Nature Reviews Rheumatology, vol. 1, no. 1, pp. 40-46, 2005.

[30] 2010, http://bloodjournal.hematologylibrary.org/content/116/ 16/2960/reply\#bloodjournal_el_591.

[31] I. Lazurova, Z. Macejova, Z. Tomkova et al., "Severe limb necrosis: primary thrombotic microangiopathy or "seronegative" catastrophic antiphospholipid syndrome? A diagnostic 
dilemma," Clinical Rheumatology, vol. 26, no. 10, pp. 1737-1740, 2007.

[32] A. S. Janoff and J. Rauch, "The structural specificity of antiphospholipid antibodies in autoimmune disease," Chemistry and Physics of Lipids, vol. 40, no. 2-4, pp. 315-332, 1986.

[33] Y. Shoenfeld, G. Twig, U. Katz, and Y. Sherer, "Autoantibody explosion in antiphospholipid syndrome," Journal of Autoimmunity, vol. 30, no. 1-2, pp. 74-83, 2008.

[34] S. Sciascia, G. Sanna, V. Murru, M. A. Khamashta, and M. L. Bertolaccini, "Validation of a commercially available kit to detect anti-phosphatidylserine/prothrombin antibodies in a cohort of systemic lupus erythematosus patients," Thrombosis Research, vol. 133, no. 3, pp. 451-454, 2014.

[35] S. Sciascia, G. Sanna, V. Murru, D. Roccatello, M. A. Khamashta, and M. L. Bertolaccini, "Antiprothrombin (aPT) and antiphosphatidylserine/prothrombin (aPS/PT) antibodies and the risk of thrombosis in the antiphospholipid syndrome. A systematic review," Thrombosis and Haemostasis, vol. 111, no. 2, pp. 354-364, 2014. 


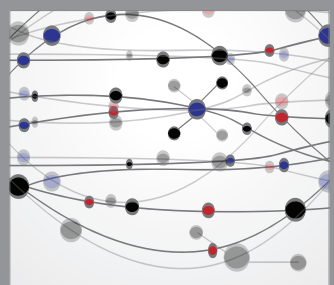

The Scientific World Journal
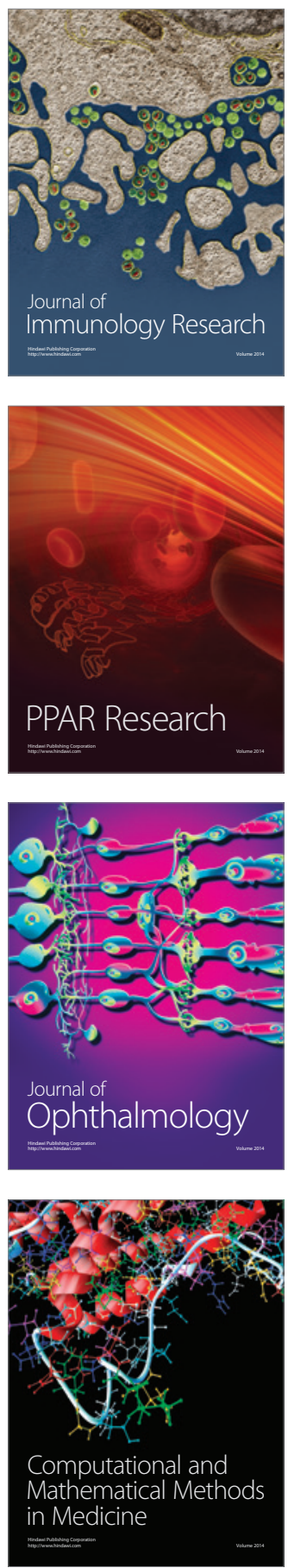

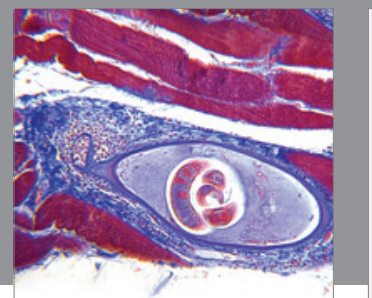

Gastroenterology

Research and Practice
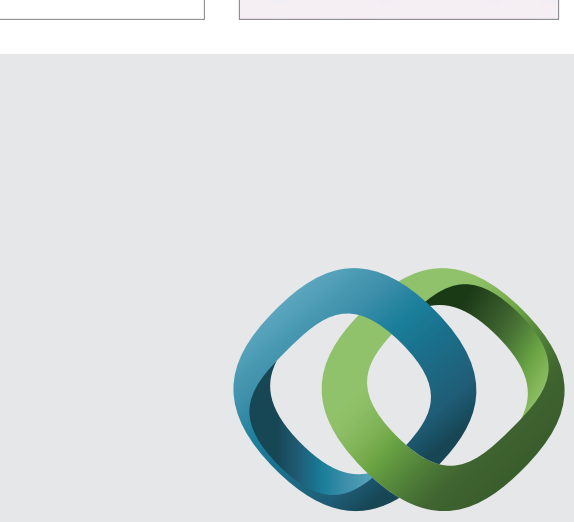

\section{Hindawi}

Submit your manuscripts at

http://www.hindawi.com
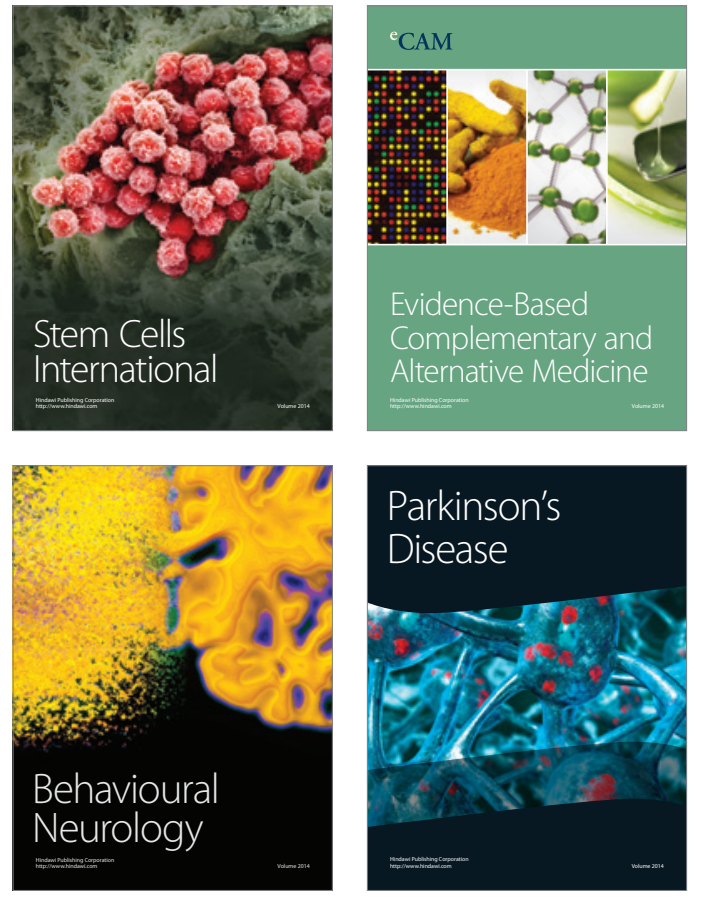
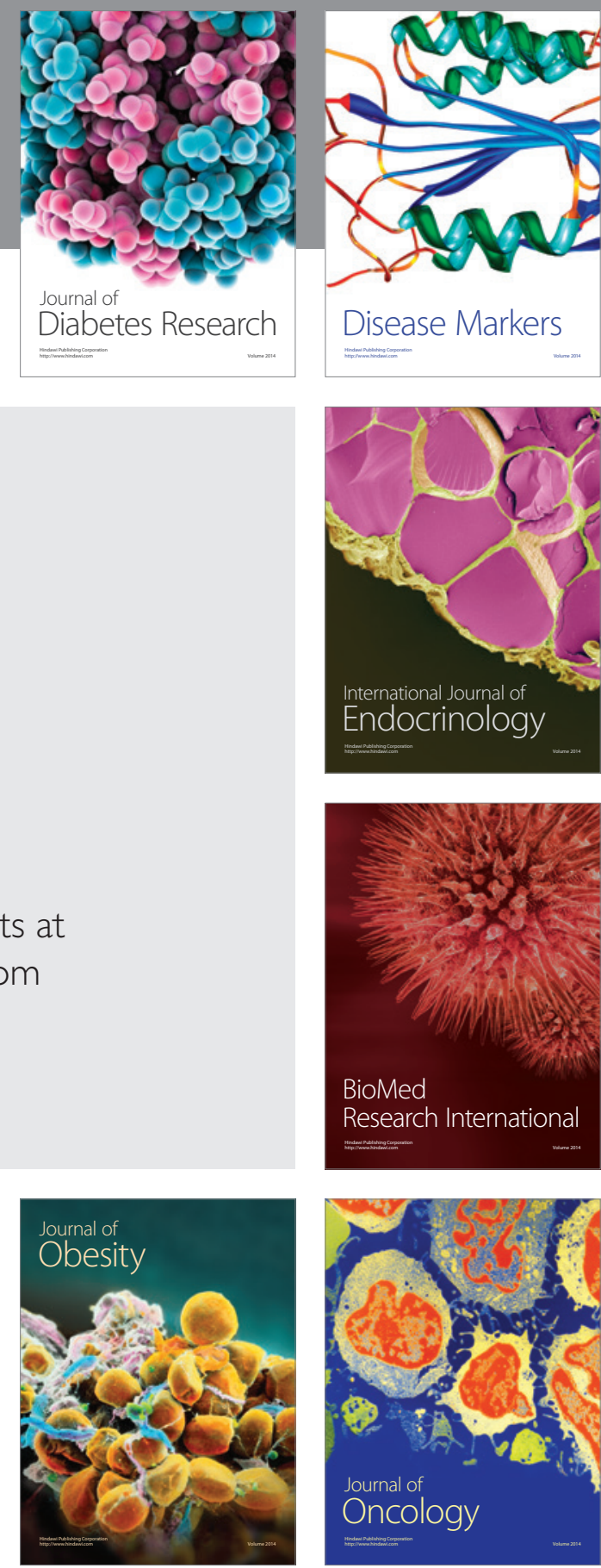

Disease Markers
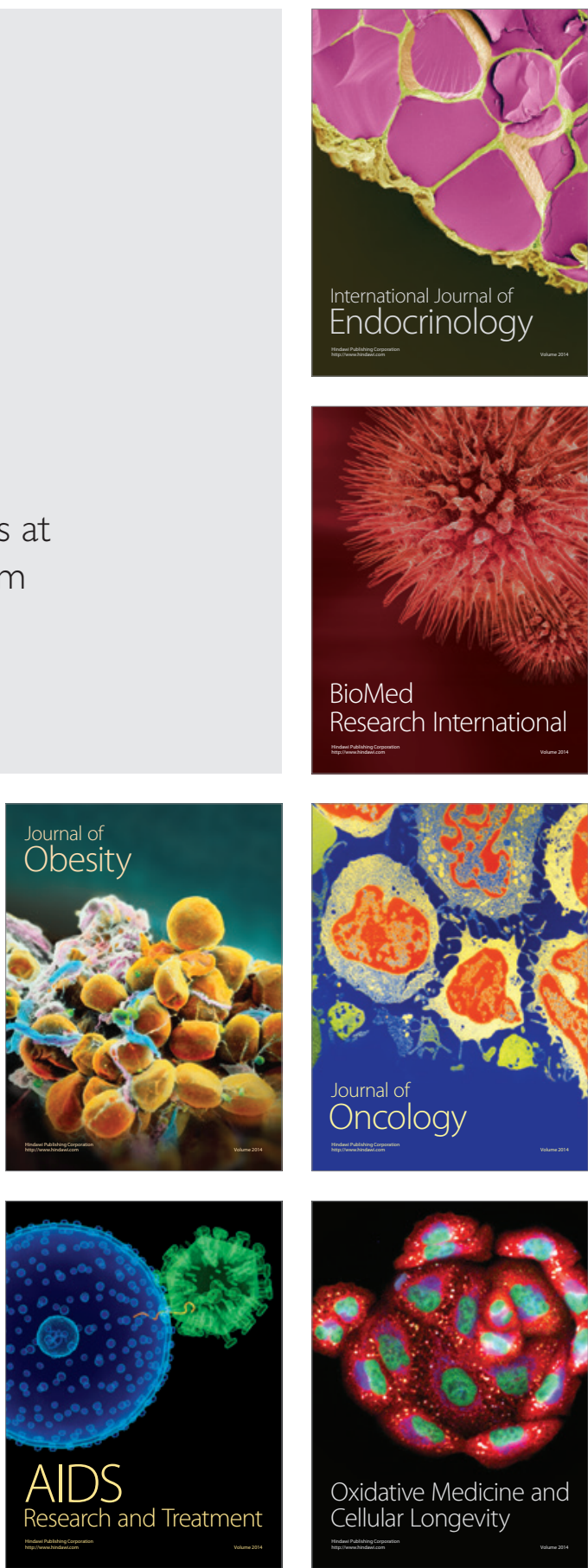